Ann. Biol. anim. Bioch. Biophys., 1978, 18 (5), 1129-1139.

\title{
Phosphatases alcalines intestinales ef composés glucidiques dans leurs rapports avec l'absorption du calcium
}

\author{
par Yronne DUPUIS, Alice DIGAUD, P. FOURNIER \\ Laboratoire du Métabolisme minéral des Mammifères, E.P.H.E. *, \\ Physiologie, Foculté de Phormacie, Tour E2, 92290 Châtenoy-Malobry.
}

\begin{abstract}
Summary. Intestinal calcium absorption and alkaline phosphatose activity : Effects of Larabinose and sorbitol.

Sorbitol action upon calcium absorption and alkaline phosphatase activity was studied in rat in situ loops at various levels of the small intestine ; its influence on both functions increased progressively from duodenum to ileum. In ileal loop, calcium absorption responded intensely to sorbitol presence but hardly at all to L-arabinose. The same carbohydrate specificity was observed with regard to ileal alkaline phosphatase. When phosphatase activity was measured from the amount of the two released molecules, using esterphosphate hydrolysis, there was always a phosphate deficit when a carbohydrate was present. This deficit was related to the phosphate transphosphorylation of the carbohydrate. The effect of a possible phosphate fixation upon the carbohydrate is discussed. This new compound would be able to postpone calcium precipitation or to lengthen the action of a calcium transfer mechanism in which the phosphatase may play a role.
\end{abstract}

En ce qui concerne l'absorption du calcium, l'existence d'un état de safuration lorsque la concentration du calcium est élevée (Cramer ef Dueck, 1962), le fait que cette absorption soit modulable par les glucides qui l'activent et par les phosphates qui l'inhibent selon des modalités précises, semblent indiquer qu'un mécanisme à caractère enzymatique est en cause (Fournier et Dupuis, 1975).

Parmi diverses enzymes caractérisant l'activité digestive des microvillosités de l'entérocyte, la phosphatase alcaline (EC 3.1.3.1) est la seule dont le fonctionnement est activé par des glucides et inhibé par des phosphates, ces composés modifiant le fonctionnement de l'enzyme dans le sens où ils modifient l'absorption du calcium (Dupuis ef al., 1976a).

Dans ce travail, nous confrontons dans divers segments de l'intestin grêle du rat les effets du L-arabinose et du sorbitol sur l'absorption du calcium ainsi que sur l'activité des phosphatases alcalines. Le mécanisme de l'intervention de l'enzyme à l'égard du glucide fera l'objet d'une étude particulière.

* ERA au CNRS no 572, Molécules, sites et transfert intestinal. 


\section{Matériel et méthodes.}

1. Animaux et protocole expérimental. - Les rats utilisés sont des mâles Wistar (souche Commentry) ; ils proviennent de l'élevage du Laboratoire où cette souche se reproduit en consanguinité depuis 1949. Ils reçoivent le régime de Randoin et Causeret (1947). On emploie des rats âgés de deux mois ou de six mois mis à jeûn depuis la veille. Sur le rat de deux mois on prépare, sous anesthésie légère à l'éther, une seule anse à quatre niveaux de l'intestin grêle : le duodénum, entre le pylore ef l'angle de Treitz; le jéjunum proximal, $12 \mathrm{~cm}$ à partir de l'angle de Treitz; le jéjunum moyen, $12 \mathrm{~cm}$ mesurés en amont d'un point situé à $30 \mathrm{~cm}$ de la jonction iléo-cæcale; l'iléum distal, $12 \mathrm{~cm}$ juste en amont de cette jonction. Pour le rat de six mois, une anse de $15 \mathrm{~cm}$ est réalisée soit sur le jéjunum proximal, soit sur l'iléum distal. Dans l'anse on injecte $1 \mathrm{ml}$ d'une solution de $\mathrm{CaCl}_{2} 10 \mathrm{mM}$ additionnée de $0,1 \mu \mathrm{Ci}$ de ${ }^{45} \mathrm{Ca}$. Pour certains essais cette solution contient en plus du L-arabinose ou du sorbitol à des concentrations allant de 10 à $200 \mathrm{mM}$. Selon l'expérience les rats sont sacrifiés deux ou quatre heures après l'injection.

Les solutions employées dont la teneur en glucide est variable ne sont pas isotoniques. Mais nous avons vérifié que la tonicité de la solution injectée dans l'anse n'influence l'absorption du calcium que si l'expérience ne dure que quelques minutes ou si la solution est hypertonique. Ce qui, présentement, n'est pas le cas.

2. Dosages ef mesures. - Chaque anse est prélevée en totalité, contenant ef contenu ; elle est minéralisée au four à 5500. Les cendres sont reprises par $\mathrm{HNO}_{3}$ concentré ef chaud. Après dilution, la radioactivité est mesurée par scintillation liquide. On déduit de cette mesure le coefficient d'absorption de ${ }^{45} \mathrm{Ca}$ en appliquant la formule :

$$
\frac{{ }^{45} \mathrm{Ca} \text { injecté }-{ }^{45} \mathrm{Ca} \text { de l'anse }}{{ }^{45} \mathrm{Ca} \text { injecté }} \times 100 \text {. }
$$

On prélève aussi un fémur dont on détermine la radioactivité. On obtient ainsi, selon Wasserman ef al. (1956) une autre expression de l'absorption de ${ }^{45} \mathrm{Ca}$. La teneur en ${ }^{45} \mathrm{Ca}$ du plasma est estimée sur le sang prélevé au moment du sacrifice.

Dans l'une des expériences, les anses n'ont pas été minéralisées afin de procéder au dosage du sorbitol. Le contenu de l'anse est déféqué par $\mathrm{ZnSO}_{4}$ et $\mathrm{OHNa}$ selon Somogyi (1930). Après oxydation périodique du sorbitol, l'aldéhyde formique produit est dosé par colorimétrie avec l'acide chromotropique, selon Burton (1957).

3. Préparation et essais des phosphatases. - Les phosphatases alcalines sont préparées à partir de la muqueuse de différents segments de l'intestin de rat. La préparation des enzymes est réalisée selon la technique de Saini et Done (1972) modifiée par Lafont (1975). Les principales étapes sont les suivantes : prélèvement de la muqueuse par grattage, homogénéisation, solubilisation en présence de butanol, précipitation par l'acétone, chromatographies successives sur colonnes de DEAE cellulose (DE 23 Whatman), Sephadex G 200 et DEAE cellulose.

L'activité phosphatasique des préparations ainsi obtenues est déterminée par hydrolyse, à $37^{\circ}$, du p-nitrophénylphosphate (PNPP) $10 \mathrm{mM}$, en tampon carbonatebicarbonate $30 \mathrm{mM}$, ̀̀ $\mathrm{pH}=9,8$, selon le protocole de Lafont (1975). Dans le milieu 
d'incubation, en plus du p-nitrophénol (PNP) mesuré par colorimétrie, on détermine la teneur en phosphate minéral selon la méthode de Briggs (1924). Certaines déterminations d'activité phosphatasique sont effectuées, selon les expériences, à $\mathrm{pH}=8,0$ ou $\mathrm{pH}=8,3$, en tampon véronal, $\mathrm{HCl} 0,1 \mathrm{M}$. Lorsque le milieu d'incubation contient du sorbitol, on emploie un réactif molybdique trois fois plus concentré afin d'éliminer l'interférence de l'hexitol (Ho et Pande, 1974). A la fin, les protéines sont déterminées par la méthode de Lowry et al. (1951).

\section{Résultats.}

1. Comparaison, en divers lieux de l'intestin grêle, des effets du sorbitol sur l'absorption du calcium et sur l'activité phosphatasique.

Le tableau 1 groupe les valeurs des coefficients d'absorption en divers lieux de l'intestin grêle. On observe que le sorbitol employé en solution $100 \mathrm{mM}$ augmente de plus en plus l'absorption à mesure que l'on s'éloigne du pylore. Dans le duodénum l'absorption du calcium est pratiquement totale pour la solution calcique seule, ce qui exclut toute possibilité d'action supplémentaire du composé glucidique. Au niveau du jéjunum proximal, les coefficients d'absorption ne sont pas significativement différents, avec ou sans sorbitol dans l'anse. L'augmentation due au sorbitol, déjà notable dans le jéjunum moyen $(P<0,01)$, devient très importante, surtout chez l'adulte, dans l'iléon distal $(P<0,001)$. A ce niveau, la présence de sorbitol se traduit par une augmentation de 60 p. 100 chez le jeune, de 92 p. 100 chez l'adulte, de l'absorption de ${ }^{45} \mathrm{Ca}$.

\section{TABLEAU 1}

Effets du sorbitol, à divers niveaux de l'intestin, sur l'absorption du calcium $\left({ }^{a}\right)$

\begin{tabular}{|c|c|c|c|c|c|}
\hline \multirow{2}{*}{$\begin{array}{c}\text { Age } \\
\text { (mois) }\end{array}$} & \multirow{2}{*}{$\begin{array}{l}\text { Concentration } \\
\text { sorbitol }(\mathrm{mM})\end{array}$} & \multicolumn{4}{|c|}{ Emplacement de l'anse } \\
\hline & & Duodénum & $\begin{array}{l}\text { Jéjunum } \\
\text { proximal }\end{array}$ & $\begin{array}{c}\text { Jéjunum } \\
\text { moyen }\end{array}$ & $\begin{array}{l}\text { lléum } \\
\text { distal }\end{array}$ \\
\hline $\begin{array}{l}2 \\
2\end{array}$ & $\begin{array}{r}0 \\
100\end{array}$ & $\begin{array}{c}95,0 \pm 2,1(b) \\
98,0 \pm 1,7 \\
\text { NS (c) }\end{array}$ & $\begin{array}{c}65,1 \pm 3,4 \\
74,0 \pm 4,1 \\
\text { NS }\end{array}$ & $\begin{array}{c}51,0 \pm 3,9 \\
78,5 \pm 5,9 \\
P<0,01\end{array}$ & $\begin{array}{l}39,2 \pm 2,4 \\
62,1 \pm 3,9 \\
P<0,001\end{array}$ \\
\hline $\begin{array}{l}6 \\
6\end{array}$ & $\begin{array}{r}0 \\
100\end{array}$ & & $\begin{array}{c}27,1 \pm 3,1 \\
31,1 \pm 1,8 \\
\text { NS }\end{array}$ & & $\begin{array}{l}22,1 \pm 1,4 \\
42,4 \pm 1,9 \\
P<0,001\end{array}$ \\
\hline
\end{tabular}

(a) Injection de $1 \mathrm{ml}$ de $\mathrm{CaCl}_{2} 10 \mathrm{mM}\left(+0,1 \mu \mathrm{Ci}^{45} \mathrm{Ca}\right)$ seul ou additionné de sorbitol à concentration finale $100 \mathrm{mM}$; sacrifice deux heures après.

(b) Coefficient d'absorption de ${ }^{45} \mathrm{Ca}$ (en p. 100 de l'injecté) : $\mathrm{m} \pm \mathrm{Sm}$ (8 rats).

(c) $\mathrm{P}=$ Degré de signification par rapport au lot 0 , à l'aide du test de Student, NS non significatif à 0,05 .

Nous avons recherché, chez l'adulte de six mois, quel étaił le comportement du sorbitol en cours d'expérience. Au sacrifice des rats, deux heures après l'injection, environ 80 p. $100 \mathrm{du}$ sorbitol initialement en solution $100 \mathrm{mM}$ se retrouve aussi bien 
dans l'anse jéjunale que dans l'anse iléale. Ce n'est donc pas à des différences dans l'absorption du sorbitol que peut être rapportée la disparité, selon le lieu, des effets du sorbitol, beaucoup plus efficace sur l'absorption calcique dans l'iléon que dans le jéjunum.

Cette sensibilité à l'égard du sorbitol, croissant en allant du duodénum vers l'iléon, s'observe aussi pour l'action de ce composé sur l'activité des phosphatases alcalines isolées des différentes régions de l'intestin grêle (tabl. 2). A l'examen des résultats consignés dans ce tableau, on remarque que l'activation de l'enzyme par le sorbitol, assez peu prononcée au niveau du duodénum, augmente progressivement devenant de plus en plus manifeste quand on s'approche du cæcum. Ainsi, tandis que le sorbitol, à une concentration $400 \mathrm{mM}$ dans le milieu d'incubation, n'augmente que de 28 p. 100 l'activité de la phosphatase duodénale, cette augmentation atteint pour la phosphatase iléale 72 p. 100 chez le jeune, 98 p. 100 chez l'adulte. Ces dernières valeurs sont très proches des augmentations de l'absorption du calcium consécutives de la présence dans ce même lieu, l'iléon dont la phosphatase est extraite, du même composé glucidique, le sorbitol.

\section{TABLEAU 2}

Effets du sorbitol sur les phosphatases alcalines intestinales

\begin{tabular}{cccccr}
\hline & & \multicolumn{3}{c}{ Origine des phosphatases alcalines } \\
\cline { 3 - 6 } $\begin{array}{c}\text { Age } \\
\text { (mois) }\end{array}$ & $\begin{array}{c}\text { Concentration } \\
\text { sorbitol (mM) }\end{array}$ & $\begin{array}{c}\text { Duodé- } \\
\text { num } \\
\mu \text { moles PNP libérées/min } / \mathrm{mg} \text { protéines }\left({ }^{a}\right)\end{array}$ \\
\hline & & $\begin{array}{c}\text { Jéjunum } \\
\text { moyen }\end{array}$ & $\begin{array}{c}\text { lléum } \\
\text { distal }\end{array}$ \\
2 & 0 & 1090 & & 232 & 89,0 \\
2 & 100 & 1204 & & 277 & 110,1 \\
2 & 400 & 1395 & & 335 & 152,4 \\
6 & 0 & & 166 & & 13,4 \\
6 & 400 & & 244 & & 26,6 \\
\hline
\end{tabular}

(a) Composition du substrat : PNPP $10 \mathrm{mM}, \mathrm{MgCl}_{2} 2 \mathrm{mM}$, tampon carbonate $30 \mathrm{mM}, \mathrm{pH} 9,8$ avec ou sans sorbitol ; les valeurs indiquées représentent la moyenne de trois essais.

2. Comparaison des effets du sorbitol et du L-arabinose, au niveau de l'iléon sur l'absorption du calcium et sur l'activité de la phosphatase alcaline.

Les résultats concernant les effets respectifs sur l'absorption de ${ }^{45} \mathrm{Ca}$ des deux composés glucidiques employés aux mêmes concentrations étagées sont groupés dans le tableau 3. Au niveau de l'iléon - mais il agit ailleurs -, le L-arabinose est sans action, sauf à la concentration très élevée de $200 \mathrm{mM}$. Jusqu'à une concentration de $70 \mathrm{mM}$ (laquelle pour le sorbitol conduit presque à tripler l'absorption), le L-arabinose est pratiquement inactif, les divers critères d'absorption n'étant pas significativement différents, en la présence ou en l'absence de ce glucide. A ce niveau iléal, le sorbitol est, au contraire, extrêmement efficace. Pour une concentration $10 \mathrm{Mm}$, l'absorption est augmentée de plus de 70 p. 100 . A la concentration de $200 \mathrm{Mm}$, l'augmentation esł de 200 p. 100 . Déjà pour une concentration $10 \mathrm{mM}$, le sorbitol agit de façon significative 
$(P<0,05)$ pour augmenter le coefficient d'absorption. Pour les deux autres critères employés les différences entre les valeurs obtenues avec et sans sorbitol ne deviennent significatives que pour des concentrations plus élevées. Malgré les insuffisances de cette expérience réalisée dans un tout autre but, il est aisé, en traçant la courbe des variations de l'absorption calcique en fonction de la concentration en sorbitol, de lui trouver des analogies avec les tracés représentant l'effet d'un activateur sur un fonctionnement enzymatique. Ce fait, établi avec d'autres composés glucidiques, a déjà été signalé (Dupuis et al., 1976b).

TABLEAU 3

Effets comparatifs du L-arabinose et du sorbitol sur l'absorption du calcium, en anses iléales $\left({ }^{a}\right)$

L-arabinose $\mathrm{mM}$

\begin{tabular}{|c|c|c|c|c|c|}
\hline $\begin{array}{r}{ }^{45} \text { Ca dans } 100 \mathrm{ml} \text { plasma (en p. } 100 \\
\text { de l'injecté) } \ldots \ldots \ldots \ldots \ldots \ldots \ldots \ldots . . .\end{array}$ & $\begin{array}{c}0 \\
7,6 \pm 0,4\left({ }^{b}\right)\end{array}$ & $\begin{array}{c}10 \\
8,7 \pm 1,1 \\
N S(c)\end{array}$ & ${ }^{9,4} \underset{N S}{ \pm} 1,0$ & $\begin{array}{c}70 \\
9,5 \pm 1,3 \\
\text { NS }\end{array}$ & $\begin{array}{c}200 \\
16,9 \pm 1,3 \\
P<0,001\end{array}$ \\
\hline $\begin{array}{c}\text { Coefficient d'absorption (en p. } 100 \\
\text { de l'injecté) } \ldots \ldots \ldots \ldots \ldots \ldots \ldots\end{array}$ & $27,3 \pm 1,9$ & $\underset{N S}{31,8 \pm 2}$ & $\underset{\text { NS }}{27,8 \pm 2,9}$ & $34,0 \pm \frac{ \pm}{N S} 3,1$ & $\begin{array}{l}72,3 \pm 6,1 \\
P<0,001\end{array}$ \\
\hline 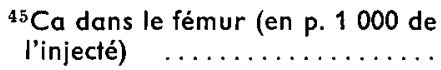 & $10,3 \pm 0,9$ & $10,8 \underset{ \pm S}{N S} 1,3$ & $10,7 \pm 1,1$ & $12,1 \underset{N S}{ \pm} 1,6$ & $\begin{array}{l}25,1 \pm 2,7 \\
P<0,001\end{array}$ \\
\hline
\end{tabular}

Sorbitol $\mathrm{mM}$

${ }^{45} \mathrm{Ca}$ dans $100 \mathrm{ml}$ plasma (en p. 100

de l'injecté) . . . . . . . . . . .

Coefficient d'absorption (en p. 100

de l'injecté) . . . . . . . . . .

${ }^{45}$ Ca dans le fémur (en p. 1000 de

l'injecté) $\ldots \ldots \ldots \ldots \ldots \ldots$

\begin{tabular}{ccccc}
\hline 0 & 10 & 30 & 70 & 200 \\
$8,6 \pm 0,9$ & $9,4 \pm 1,3$ & $9,9 \pm 0,8$ & $11,4 \pm 1,2$ & $17,3 \pm 2,1$ \\
& $N S(c)$ & NS & NS & $P<0,01$ \\
$22,9 \pm 3,1$ & $39,9 \pm 6,2$ & $50,5 \pm 4,3$ & $60,5 \pm 5,8$ & $82,0 \pm 6,4$ \\
& $P<0,05$ & $P<0,001$ & $P<0,001$ & $P<0,001$ \\
$11,7 \pm 1,3$ & $16,3 \pm 3,3$ & $19,1 \pm 2,1$ & $21,1 \pm 1,9$ & $30,3 \pm 2,4$ \\
& $N S$ & $P<0,01$ & $P<0,01$ & $P<0,001$
\end{tabular}

(a) Injection de $1 \mathrm{ml}$ de $\mathrm{CaCl}_{2} 10 \mathrm{mM}\left(+0,1 \mu \mathrm{Ci}^{45} \mathrm{Ca}\right)$, seul ou additionné d'un glucide ; sacrifice 4 heures après.

(b) $\mathrm{m} \pm \mathrm{Sm}$ ( $\mathrm{n}=8$ rats de deux mois).

(c) $\mathrm{P}=$ Degré de signification par rapport au lot 0 , calculé par le test de Student, NS non significatif à 0,05 .

Cette différence, au même niveau iléal, dans le comportement des deux composés glucidiques à l'égard de l'absorption du calcium se retrouve en ce qui concerne leur influence sur la phosphatase alcaline extraite de l'iléon. Pour des concentrations déjà élevées (200 et $500 \mathrm{mM}$ ), le L-arabinose influence peu l'activité phosphatasique, que l'essai soit réalisé à $\mathrm{pH} 8,3$ ou 9,8 et que l'activité soif jugée d'après le PNP ou le phosphate libéré (tabl. 4). Comme cela était le cas pour l'action de ce pentose sur l'absorption du calcium, il faut arriver à une forte concentration pour qu'un effet net se manifeste, et c'est dans le sens d'une inhibition. Aux deux pH auxquels l'activité de la phos- 
phatase est mesurée, on observe que le nombre de molécules de phosphate libéré est nettement inférieur à celui des molécules dePNP, environ de 30 p. 100 à pH 9,8. Notons qu'en absence de glucide dans le milieu d'incubation, les deux valeurs se correspondent assez bien.

\section{TABLEAU 4}

Effets du L-arabinose et du sorbitol sur l'activité de la phosphatase iléale $\left({ }^{a}\right)$

\begin{tabular}{|c|c|c|c|c|c|c|c|}
\hline \multirow[b]{3}{*}{$\mathrm{pl}$} & \multirow{3}{*}{$\begin{array}{c}\text { Concentration } \\
\text { en composé } \\
\text { glucidique } \mathrm{mM}\end{array}$} & \multicolumn{4}{|c|}{$\begin{array}{c}\text { Produits formés } \\
(\mu \text { moles } / \mathrm{min} / \mathrm{mg} \\
\text { protéines) }\end{array}$} & \multirow{2}{*}{\multicolumn{2}{|c|}{$\begin{array}{c}\text { Phosphate } \\
\text { transféré } \\
\frac{(1)-(2)}{(1)} \times 100\end{array}$}} \\
\hline & & \multicolumn{2}{|c|}{ PNP (1) } & \multicolumn{2}{|c|}{$\mathrm{Pi}(2)$} & & \\
\hline & & 8,3 & 9,8 & 8,3 & 9,8 & 8,3 & 9,8 \\
\hline L-arabinose & $\begin{array}{r}0 \\
200 \\
500 \\
1000\end{array}$ & $\begin{array}{l}8,8\left(^{b}\right) \\
9,4 \\
9,1 \\
8,1\end{array}$ & $\begin{array}{l}31,6 \\
26,0 \\
25,4 \\
21,2\end{array}$ & $\begin{array}{l}9,2 \\
9,2 \\
8,6 \\
6,7\end{array}$ & $\begin{array}{l}33,2 \\
27,2 \\
24,8 \\
15,1\end{array}$ & $\begin{array}{r}-4,5 \\
2,1 \\
5,5 \\
17,3\end{array}$ & $\begin{array}{r}-4,6 \\
4,6 \\
2,4 \\
28,8\end{array}$ \\
\hline Sorbitol & $\begin{array}{r}200 \\
500 \\
1000\end{array}$ & $\begin{array}{l}10,6 \\
11,5 \\
11,6\end{array}$ & $\begin{array}{l}36,6 \\
44,3 \\
46,5\end{array}$ & $\begin{array}{l}9,7 \\
8,3 \\
8,5\end{array}$ & $\begin{array}{l}25,1 \\
31,4 \\
29,1\end{array}$ & $\begin{array}{r}8,5 \\
27,8 \\
26,7\end{array}$ & $\begin{array}{l}31,4 \\
29,1 \\
37,4\end{array}$ \\
\hline
\end{tabular}

(a) Rats de deux mois.

$\left({ }^{b}\right)$ Moyenne de trois essais.

Cette disparition d'une fraction des phosphates est presque toujours observée lorsque le composé glucidique est du sorbitol. En ce qui concerne le PNP libéré, le sorbitol en augmente la quantité, aux deux $\mathrm{pH}$ et aux trois concentrations considérées. Par contre, à une exception près, la quantité de phosphate retrouvée est, par rapport aux essais réalisés en l'absence de glucide, soit inchangée, soit diminuée. Puisque, à l'inverse, la quantité de PNP est augmentée, c'est donc presque toujours un déficit de phosphate qui est constaté. Ce déficit, qui ne peut correspondre qu'à un transfert de phosphate du substrat PNPP sur le sorbitol est de l'ordre de 30 p. 100 dans cinq des six cas.

3. Etude, à divers $\mathrm{pH}$, des relations entre les phosphatases alcalines ef le sorbitol.

Cette expérimentation ne consiste plus, comme les précédentes, en une comparaison de l'action de composés glucidiques sur les phosphatases et sur l'absorption calcique. C'est un examen des relations entre ces enzymes et le sorbitol. Les résultats font l'objet du tableau 5.

La présence du composé glucidique a, selon l'origine de la phosphatase et selon le $\mathrm{pH}$, des effets variés sur l'activité des enzymes jugée d'après les moles de PNP libérées. La présence de sorbitol a pour effet d'augmenter la quantité de PNP libérée, le pourcentage d'activation de l'enzyme étant de plus en plus marqué à mesure que l'on s'approche du cæcum. Ces résultałs corroborent ceux de la première expérimen- 
tation effectuée à $\mathrm{pH} 9,8$. Il en va tout autrement à $\mathrm{pH} 8,0$ puisque la présence de sorbitol n'a aucun effet sur le PNP mesuré après action de la phosphatase iléale et que l'on note même un effet inhibant du composé glucidique sur la phosphatase duodénale.

TABLEAU 5

Effets du sorbitol sur des phosphatases alcalines duodénales et iléales, à $\mathrm{pH} 8,0$ et $9,8\left({ }^{a}\right)$

\begin{tabular}{|c|c|c|c|c|c|c|}
\hline \multirow[b]{2}{*}{$\begin{array}{c}\text { Origine } \\
\text { de la phosphatase }\end{array}$} & \multirow[b]{2}{*}{$\mathrm{pH}$} & \multirow[b]{2}{*}{$\begin{array}{c}\text { Hexitol } \\
\text { ajouté } \\
(\mathrm{mM})\end{array}$} & \multicolumn{3}{|c|}{ Activités } & \multirow{2}{*}{$\begin{array}{c}\begin{array}{c}\text { Phosphate } \\
\text { transféré }\end{array} \\
\frac{(1)-(2)}{(1)} \times 100\end{array}$} \\
\hline & & & $\begin{array}{l}\text { Phospha } \\
\text { ( } \mu \text { moles } \\
\text { proté } \\
\text { PNP (1) }\end{array}$ & $\begin{array}{l}\text { asique } \\
\mathrm{nin} / \mathrm{mg} \\
\text { nes) } \\
\mathrm{Pi}(2)\end{array}$ & $\begin{array}{l}\text { Transphos- } \\
\text { phorylante } \\
\text { (1) - (2) }\end{array}$ & \\
\hline Duodénum & $\begin{array}{l}8,0 \\
9,8\end{array}$ & $\begin{array}{r}0 \\
500 \\
0 \\
500\end{array}$ & $\begin{array}{l}404\left(^{b}\right) \\
354 \\
1090 \\
1450\end{array}$ & $\begin{array}{r}360 \\
241 \\
1000 \\
901\end{array}$ & $\begin{array}{r}44 \\
113 \\
90 \\
549\end{array}$ & $\begin{array}{r}11 \\
32 \\
8 \\
38\end{array}$ \\
\hline lléum & $\begin{array}{l}8,0 \\
9,8\end{array}$ & $\begin{array}{r}0 \\
500 \\
0 \\
500\end{array}$ & $\begin{array}{r}42 \\
42 \\
91 \\
156\end{array}$ & $\begin{array}{l}39 \\
29 \\
82 \\
82\end{array}$ & $\begin{array}{r}3 \\
13 \\
9 \\
71\end{array}$ & $\begin{array}{l}7 \\
31 \\
10 \\
46\end{array}$ \\
\hline
\end{tabular}

(a) Rats de deux mois.

$(b)$ Moyenne de trois essais.

Pour ce qui est des phosphates, les résultats sont bien différents. $\mathrm{A} \mathrm{pH} \mathrm{9,8,} \mathrm{la}$ présence de sorbitol ne modifie pas la quantité de phosphate libérée par la phosphatase iléale, tandis que cette quantité diminue pour la duodénale. $\mathrm{A} \mathrm{pH} \mathrm{8,0,} \mathrm{on} \mathrm{observe}$ toujours, par rapport aux témoins, une diminution importante des quantités de phosphate retrouvées. L'effet du sorbitol sur l'activité des phosphatases se traduisant par des quantités de PNP augmentées ou inchangées tandis que les quantités de phosphate sont toujours nettement diminuées, le déficit en phosphate est toujours important. Ainsi, en présence de sorbitol, les deux valeurs par lesquelles on mesure l'hydrolyse du PNPP, valeurs qui déjà présentent une différence inexpliquée de l'ordre de 10 p. 100 en l'absence du glucide, sont en sa présence dans tous les cas décalées d'environ 40 p. 100. Ce dernier phénomène montre que le pouvoir transphosphorylant de l'enzyme est, en tous lieux de l'intestin et pour de larges intervalles de $\mathrm{pH}$, le phénomène constant qu'il convient de considérer.

\section{Discussion.}

Parmi divers points traités dans ce travail certains doivent être soulignés, examinés ou seulement abordés.

1. Corrélations en série entre activité phosphatasique et absorption du calcium.

L'emploi des glucides conduit à souligner le parallélisme des variations de l'absorption calcique ef de l'activité phosphatasique. La corrélation qui s'établissait 
d'après la propriété commune que possèdent certains glucides d'activer l'une et l'autre de ces fonctions devient de plus en plus étroite quand, à cette relation entre fonctions, se superposent des spécificités de lieu. Depuis le pylore jusqu'au cæcum, on note l'augmentation progressive des effets du sorbitol aussi bien sur l'absorption du calcium que sur l'activité des phosphatases. De plus, pour un lieu donné, on note une spécificité glucidique, l'absorption n'étant augmentée que par celui des glucides qui active les phosphatases extraites en ce lieu.

On peut néanmoins objecter que les essais sur l'absorption du calcium ont été réalisés à des concentrations glucidiques différentes de celles employées pour examiner l'activité des phosphatases. L'effet activant du sorbitol sur le pouvoir hydrolasique de la phosphatase est bien décelable à une concentration $40 \mathrm{mM}$ (Dupuis et al., 1977). On l'observe dans les essais réalisés à $\mathrm{pH}=9,8$ aux concentrations de sorbitol $100 \mathrm{mM}$ (tabl. 2) et $200 \mathrm{mM}$ (tabl. 4). Mais dans ce dernier cas, à pH 8,3, l'effet transphosphorylant n'apparaît pas. II faut atteindre une concentration $400 \mathrm{mM}$ en sorbitol pour obtenir une augmentation d'activité de la phosphatase iléale comparable à l'augmentation de l'absorption du calcium que l'on observe, dans l'iléon, sous l'effet d'une concentration $100 \mathrm{mM}$. Une telle situation n'est pas exceptionnelle : ainsi pour observer une action du lactose sur la fixation de ${ }^{45} \mathrm{Ca}$ par une anse iléale retournée, une concentration de $160 \mathrm{mM}$ dans le milieu d'incubation est nécessaire ; une concentration $40 \mathrm{mM}$ est sans action. Pourtant à cette dernière concentration, ce glucide agit efficacement, in vivo, sur l'absorption du calcium. Il faut donc un rapport de 1 à 4 entre les concentrations à employer in vivo et in vitro pour que le lactose manifeste ses effets. Ce rapport est celui qui, présentement, offre les conditions optimales pour étudier l'action du sorbitol in vivo sur l'absorption du calcium (100 mM) et in vitro sur l'activité phosphatasique $(400 \mathrm{mM})$. D'ailleurs cette solution $100 \mathrm{mM}$ en sorbitol, inférieure à la concentration $200 \mathrm{mM}$ pour laquelle, à pH 8,3, la phosphatase iléale ne peut pas phosphoryler cet hexitol (tabl. 4), suscite cependant, dans la muqueuse et le contenu de l'anse iléale où on l'injecte la formation de quantités notables de sorbitol phosphate (Digaud, travaux en cours).

Ces nouvelles corrélations fondées sur des effets parallèles des composés glucidiques sur l'absorption du calcium et sur l'activité des phosphatases alcalines intestinales viennent s'ajouter à d'autres observations faites dans des circonstances variées. Holdsworth (1970) montre chez le poulet que, sous l'effet de l'administration de vitamine D, l'activité de cerlaines enzymes des bordures en brosses de l'entérocyte- ATPase, phosphatase alcaline, pyrophosphatase - augmente, ainsi que l'absorption du calcium. Mais les activités enzymatiques ef l'absorption ne sont pas étudiées au même niveau de l'intestin. Toujours sur le poulet rachitique, Haussler ef al. (1970), Norman et al. (1970) relèvent sous l'effet de la vitamine D la correspondance entre l'augmentation de l'activité de la phosphatase et celle de l'absorption calcique. L'administration de glucocorticoïdes fait varier simultanément ces deux fonctions et établissent entre elles une nouvelle corrélation (Krawitt, 1972). Chez le jeune rat carencé en calcium, on observe au niveau du duodénum seulement une augmentation de la capacité d'absorption de cet élément et de l'activité phosphatasique (Krawitt et al., 1973). Chez le rat axénique, l'absorption du calcium et l'activité des phosphatases alcalines intestinales sont plus élevées que chez l'animal conventionnel (Reddy, 1972). 
Par comparaison des électrophorèses réalisées sur les protéines des bordures en brosse de poulets rachitiques venant de recevoir ou non des métabolites de la vitamine $D_{3}$, on observe après traitement un changement d'emplacement d'une protéine à forte activité phosphatasique. Sous l'effet du $1-25(\mathrm{OH})_{2} D_{3}$ ce changement s'opère dans les six heures, temps correspondant à celui de l'augmentation de l'absorption du calcium, tandis que les augmentations d'activité phosphatasique et de teneur en CaBP se révèlent plus tardivement. L'extrait de muqueuse duodénale présente aussi précocement une aptitude accrue à lier le calcium. Ce déplacement dans la position de la protéine correspondrait à une augmentation de poids par fixation de CaBP sur la phosphatase. Un tel édifice moléculaire interviendrait dans le transfert du calcium (Moriuchi et De Luca, 1976). Quand, en cours d'expérience, on défecte chez un rat des dispositions anormalement élevées à l'absorption du calcium, ce rat présente aussi une activité phosphatasique intestinale élevée tandis que d'autres enzymes localisées dans les bordures en brosse et les phosphatases d'autres tissus présentent des valeurs normales (Fournier et al., 1977).

Sans rien préjuger des rapports exacts entre l'enzyme et le minéral, il devient de plus en plus vraisemblable que la phosphatase alcaline est en relation avec les échanges de calcium, notamment au niveau du transfert intestinal.

\section{Sur l'effet transphosphorylant de la phosphatase sur le glucide.}

L'effet transphosphorylant des phosphatases alcalines ou acides, connu depuis longtemps, a fait l'objet d'importants travaux (Axelrod, 1948 ; Meyerhoff et Green, 1950). Ainsi a été établie la capacifé que possèdent ces enzymes de fixer sur les fonctions hydroxylées de nombreux composés la moitié au maximum des molécules de phosphate qu'elles libèrent à partir d'un ester-phosphate, cette transphosphorylation s'effectuant mieux sur un hexitol que sur un ose (Morton, 1958). Ainsi le sorbitol, meilleur accepteur de phosphate, est, comme le montre la présente expérimentation, le composé glucidique qui augmente le mieux l'absorption du calcium. En fait, le mécanisme par lequel les phosphatases alcalines interviennent dans les métabolismes reste inconnu en dépit du nombre considérable de travaux dont cette enzyme si abondante et répandue a fait l'objet. La raison de cette ignorance n'est peut-être pas étrangère au fait que l'essai de ses propriétés a pratiquement toujours été effectué à un $\mathrm{pH}$ voisin de 10 et le plus souvent au moyen de substrats choisis pour la commodité de leur emploi, toutes circonstances fort éloignées des conditions physiologiques dans lesquelles l'enzyme exerce ses activités. Le mécanisme par lequel le glucide augmente l'absorption du calcium n'est pas précisé. Selon Armbrecht ef Wasserman (1976) le glucide augmenterait la perméabilité de la membrane au calcium mais la formation éventuelle d'un complexe entre le glucide et le calcium ne pourrait être retenue.

Devant tant d'incertitudes sur les rôles que peuventtenị les phosphatases et certains glucides, notamment en ce qui concerne leurs relations avec l'absorption du calcium, les présentes observations pourraient être instructives. Diverses expérimentations ont conduit à élaborer un mécanisme impliqué dans le transfert du calcium, mécanisme auquel pourraient participer la phosphatase, les glucides, les phosphates (Dupuis ef al., 1977). Or les résultats des précédentes expériences permettent de relier fonctionnellement ces trois composés, en même temps qu'ils autorisent à attribuer un 
rôle plausible à l'enzyme ef au glucide. Normalement la phosphatase alcaline hydrolyse des esters-phosphates. Classiquement son activité se trouve contrariée par les phosphates qu'elle libère. Aussi conçoit-on qu'en présence de glucide à qui elle confère un rôle d'accepteur de phosphate, l'inhibition de l'enzyme se trouve diminuée.

3. Relations éventuelles entre l'action de la phosphatase sur le glucide ef l'absorption du calcium.

Sur ce point capital, on ne saurait rien affirmer. Tout au plus peut-on fournir quelques éléments nécessaires à son étude, dans la mesure où l'effet transphosphorylant de la phosphatase sur le glucide serait à considérer.

Le composé glucidique augmente l'absorption du calcium en prolongeaní sa durée (Lengemann et al., 1959). De fait, en anse iléale, l'absorption idu calcium de la solution qui ne contient pas de glucide est terminée en une demi-heure. A ce moment le taux d'absorption du calcium de la solution qui, de plus, contient un glucide n'est pas sensiblement différente de celui de la précédente. Mais, du fait de la présence du glucide, l'absorption du calcium se prolonge tandis que dans la solution non glucidique l'absorption calcique s'arrête. Pourquoi l'absorption cesse-t-elle? Est-ce le calcium de la solution qui, insolubilisé éventuellement sous forme de phosphate, n'est plus absorbable?

Cette insolubilisation précoce du minéral, retardée en présence de glucides, pourrait rendre compte du fait que ces composés augmentent aussi l'absorption de minéraux dont les phosphates sont peu solubles en milieu intestinal, tels ceux de $\mathrm{Fe}$, de $\mathrm{Mn}$, de $\mathrm{Zn}$ et de Co. Mais un accroissement de solubilité semble difficilement en cause dans le fait que le lactose qui augmente beaucoup l'absorption du calcium, augmente aussi celle du taurocholate de sodium (Riottot ef al., 1977). Est-ce plutôt le mécanisme de membrane qui est inhibé ? La réponse à ces questions peut être importante.

De toute façon le mécanisme proposé où le glucide joue un rôle d'accepteur de phosphate peut rendre compte de l'une ou l'autre de ces éventualités. Par la formation d'un ose-phosphate de calcium plus soluble que le phosphate correspondant, la présence du glucide peut différer la précipitation du calcium. En retardant l'inhibition de la phosphatase alcaline par le phosphate, la présence du glucide peut permettre à un mécanisme de membrane auquel l'enzyme participerait de fonctionner plus longtemps.

Reçu en décembre 1977. Accepté en mars 1978.

\section{Références}

ARMBRECHT H. J., WASSERMAN R. H., 1976. Enhancement of ++ Ca uptake by lactose in the rat small intestine. J. Nutr., 106, 1265-1271.

AXELROD B., 1948. A study of the mechanism of " phosphotransferase 》 activity by use of *P. J. biol. Chem., 176, 296-298.

BRIGGS A. P., 1924. Some application of the colorimetric phosphate method. J. biol. Chem., 59, 255264.

BURTON R. M., 1957. The determination of glycerol and dihydroxyacetone, 246-248. In COLOWICK S. P., KAPLAN W. O., Methods in enzymology, vol, 3, Acad. Press. N. Y. 
CRAMER C. F., DUECK J., 1962. In vivo transport of calcium from healed Thiry-Vella fistula in dogs. Am. J. Physiol., 202, 161-164.

DUPUIS Y., FOURNIER P., 1976a. Etude, en anses intestinales in situ, de la modulation par un glucide et par un phosphate de l'absorption du calcium. C. R. Soc. Biol., 170, 304-309.

DUPUIS Y., FOURNIER A., FOURNIER P., 1976b. Etude, au niveau de la muqueuse jéjunale, des effets de divers composés glucidiques sur l'activité de quelques enzymes localisées dans les bordures en brosse. Accroissement d'activité phosphatasique. C. R. Soc. Biol., 170, 739-744.

DUPUIS Y., DIGAUD A., FONTAINE N., 1977. Etude des effets du sorbitol sur l'activité des phosphatases alcalines isolées des diverses régions de l'intestin grêle du rat. C. R. Soc. Biol., 171, 294302.

FOURNIER P., DUPUIS Y., 1975. La modulation de l'absorption intestinale du calcium. J. Physiol., Paris, 70, 479-491.

FOURNIER P., FONTAINE N., FOURNIER A., 1977. Nouvelle corrélation entre l'absorption du calcium et l'activité des phosphatases alcalines intestinales. Ann. Nutr. Alim., 31, 277-290.

HAUSSLER M. R., NAGODE L. A., RASMUSSEN H., 1970. Induction of intestinal brush border alkaline phosphatase by vitamine D and identity with Ca-ATPase. Nature, London, 228, 1190-1201.

HO C. H., PANDE S. V., 1974. Orthophosphate determination : interference by mannitol and sorbitol. Analyt. Biochem., 60, 443-446.

HOLDSWORTH E. S., 1970. The effect of vitamine D on enzyme activities in the mucosal cells of the chick small intestine. J. Memb. Biol., 3, 43-53.

KRAWITT E. L., 1972. The role of intestinal transport proteins in cortisone mediated suppression of calcium absorption. Biochim. biophys. Acta, 274, 179-188.

KRAWITT E. L., STUBBERT A. S., ENNIS P. H., 1973. Calcium absorption and brush border phosphatases following dietary calcium restriction. Am. J. Physiol., 224, 548-551.

LAFONT J., 1975. Rôle métabolique des phosphatases alcalines au niveau de l'entérocyte. Thèse Doct. Sci. Phys., Montpellier.

LENGEMANN F. W., WASSERMAN R. H., COMAR C. L., 1969. Studies on the enhancement of radiocalcium and radiostrontium absorption by lactose in the rat. J. Nutr., 68, 443-456.

LOWRY O., ROSEBROUGH N., FARR A., RANDALL R., 1951. Protein measurement with the Folin phenol reagent. J. biol. Chem., 193, 265-275.

MEYERHOFF O., GREEN H., 1950. Synthetic action of phosphatase. 11. Transphosphorylation by alkaline phosphatase in the absence of nucleotides. J. biol. Chem., 183, 377-390.

MORIUCHI S., DE LUCA H. F., 1976. The effects of vitamin $D_{3}$ metabolites on membrane proteins of chick duodenal brush borders. Arch. Biochem. Biophys., 174, 367-372.

MORTON R. K., 1958. The phosphotransferase activity of phosphatases. Biochem. J., 70, 139-150.

NORMAN A. W., MIRCHEFF A. K., ADAMS T. H., SPIELVOGEN A., 1970. Studies on the mechanism of action of calciferol. Ill. Vitamin D mediated increase of intestinal brush border alkaline phosphatase activity. Biochim. biophys. Acfo, 215, 348-359.

RANDOIN L., CAUSERET J., 1947. Constitution d'un régime alimentaire équilibré pour les jeunes rats blancs destinés au dosage des vitamines $A$ et $D$ par les méthodes biologiques. Bull. Soc. Sci. Hyg. Alim., 35, 14-22.

REDDY B. S., 1972. Studies on the mechanism of calcium and magnesium absorption in germfree rats. Arch. Biochem. Biophys., 149, 15-21.

RIOTTOT M., SACQUET E., LEPRINCE C., MEJEAN C., 1977. Effect of dietary lactose on the absorption of sodium taurocholate in the rat ileum. Nutr. Rep. infernat., 15, 421-427.

SAINI P. K., DONE J., 1972. The diversity of alkaline phosphatases from rat intestine isolation and purification of the enzyme(s). Biochim. biophys. Acta, 258, 147-153.

SOMOGYIM., 1930. A method for the preparation of blood filtrates for the determination of sugar. J. biol. Chem., 86, 655-663.

WASSERMAN R. H., COMAR C. L., NOLD M. M., 1956. The influence of aminoacids and other organic compounds on the gastrointestinal absorption of calcium ${ }^{45}$ and strontium ${ }^{89}$ in the rat. J. Nutr., 59, 371-383. 